\title{
Erratum to: Neighborliness of marginal polytopes
}

\section{T. Kahle}

\section{Beitr Algebra Geom (2010) 51:45-56}

Regrettably my paper "Neighborliness of marginal polytopes" contains mistakes in the remark "Maximizing Multiinformation": Corollary 1 there does not include any theorem of the given reference [3], its statement is only a special case of Theorem 5.1 in [3]. Moreover, Corollary 2 does not imply Corollary 1, for which reason it is no longer as general as I claimed. I apologize for this inexactness and wish to thank Nihat Ay and Andreas Knauf for pointing this out to me.

\section{Reference}

3. Ay, N., Knauf, A.: Maximizing multi-information. Kybernetika 42, 517-538 (2006)

The original article can be found under http://www.emis.de/elibm/journals/index.html.

T. Kahle $(\varangle)$

Max Planck Institute for Mathematics in the Sciences,

Inselstraße 22, 04103 Leipzig, Germany

e-mail: kahle@mis.mpg.de 